\title{
Effects of transcriptional factor BXIN_0799 on transcription of genes related to invasion and asexual development of Babesia sp. Xinjiang
}

\author{
Jinming Wang ( $\sim$ wjm0403@caas.cn ) \\ Lanzhou Veterinary Research Institute

\section{Yijun Chai} \\ Lanzhou Veterinary Research Institute

\section{Youquan Li} \\ Lanzhou Veterinary Research Institute \\ Jianxun Luo \\ Lanzhou Veterinary Research Institute \\ Hong Yin \\ Lanzhou Veterinary Research Institute \\ Guiquan Guan \\ Lanzhou Veterinary Research Institute
}

\section{Research Article}

Keywords: transcription factor, Babesia, asexual development, transcriptional regulation, ovine babesiosis

Posted Date: January 31st, 2022

DOI: https://doi.org/10.21203/rs.3.rs-1274619/v1

License: @ (i) This work is licensed under a Creative Commons Attribution 4.0 International License. Read Full License 


\section{Abstract}

Background: Transcription factors (TF) of Apicomplexan bearing AP2 DNA-binding domains (ApiAP2s) are identified in Babesia sp. Xinjiang (BXj) and found to perform regulatory functions in parasite development. However, the functions of specific TFs in Bxj remain unknown.

Methods: Here, homologous recombination technology was employed to generate a mutant strain in which BXIN_0799 gene was disrupted. A plasmid was constructed: DNA fragments of ef $1 a-B 5^{\prime}$ non-coding region (ef1a-NR) and rap3' terminal region (rap-TR) were amplified from $B \times j$ genomic DNA, and the blasticidin-S deaminase (BSD) gene was amplified from plasmid pgfp-bsd-ef. $20 \mu \mathrm{g}$ of circular plasmid was transfected into Bxj merozoites to disrupt BXIN_0799 gene.

Results: Transcriptome analyses revealed that 282 genes were down regulated, and 95 of these were proteins of unknown function. These downregulated genes are involved in critical processes of asexual development of $B x j$, including transcriptional regulation, humoral immune response (Variant erythrocyte surface antigen-1, VESA1), and host cell invasion (Rhoptry neck protein 2, Rhoptry-associated protein 1). We confirmed that BXIN_0799 performed non-essential functions in BXj asexual development (red blood cell stage), as the mutant strain reflected a slightly reduced growth rate in vitro culture system. Compared to wild type (WT), the growth rate of mutant strain was decreased by approximately $15 \%$.

Conclusions: In this study, we illustrate the regulation function of BXIN_0799 in Bxj asexual development. A slight influence was observed in RBC stage development. 95 down-regulated genes code unknown functional proteins. Whether these products contribute to gametocytogenesis in the RBC stage, sexual development, and even blocking transmission is need to further study.

\section{Background}

Phylum Apicomplexa is composed of obligate intracellular parasites, including Plasmodium falciparum (primary causative agent of human malaria), Toxoplasma gondii (the major cause of human and animal toxoplasmosis), Cryptosporidium parvum (responsible for cryptoridiosis), and Babesia spp. (animals and zoonotic pathogens). These devastating pathogens pose significant risks to public health and have caused huge economic losses in agricultural production worldwide [1]. Since the first case of Babesia species was reported in 1888, more than 100 species have been identified [2]. The Babesia genus also consists of important public health and livestock species, such as $B$. microti, $B$. divergens, and B. bovis. Most of our knowledge of this genus pertains to $B$. microti and $B$. bovis, which are responsible for the majority of Babesia species infections in humans and cattle, respectively, throughout the world. However, compared with Plasmodium spp. and Toxoplasma spp., which are responsible for the majority of cases of human death, Babesia spp., which account for relatively few cases of human babesiosis, have not been extensively studied $[3,4,5]$. Recently, a novel Babesia species, experimentally transmitted by Hyalomma anatolicum anatolicum, was named Bxj and isolated from a sheep bitten by Rhipicephalus sanguineus and $H$. anatolicum anatolicum [6, 7]. Molecular and serological investigations revealed that $B x j$ was wide distribution across China, suggesting a serve threat to sustainable development of sheep industry in China [8]. The lack of effective vaccine and drug resistance have made the situation worse. Limited information regarding $B x j$ biological feature, and parasite-host and parasite-vector interaction, is available, which hinder the drug development and vaccine exploration. Comprehensively understanding of Bxj development and transmission mechanisms will provide valuable information for controlling even to eliminating this disease.

Due to the rapid development of genomic sequencing, increasing numbers of Apicomplexan parasites genome are available; comparative genomic analysis can be performed to identify virulence factors determining pathogenicity and gene families involved in host cell invasion, and gain insight into adaptive evolution to hosts $[9,10,11]$. Gene sets of AP2 are of the most noticeable TF gene families in Apicomplexan, which contain one or several DNA-binding domain(s). However, Apicomplexans lack of specific TFs with DNA binding domains that are closely related to those in TFs families found in other eukaryotic super-kingdoms, such as TATA box-binding protein (TBP), transcription factor IIB (TFIIB), transcription factor IIE (TFIIE), and Multiprotein bridging factor 1 (MBF1) $[12,13,14]$. Several of TFs (Api-AP2 gene family) were identified in $P$. falciparum and $C$. 
parvum, but they lack conserved DNA binding domains of specific TFs identified in eukaryote. Performing similar functions as that of Api-AP2 gene family, HMG and Myb genes were also identified in apicomplexa parasites [15]. The TF families ApiAP2 in Apicomplexan parasites is comprised of 27 members in $P$. falciparum, and its members perform multiple functions in host cell invasion and parasites survival. PfAP2-G and PfAP2-I play roles in red blood cell (RBC) invasion [16]. PfAP2-G activates gametocyte gene and PfAP2-I may play an important role in parasite survive [17, 18, 19]. PfAP2-EXP is involved in regulating multi-gene families, including rif, stevor, pfmc-2tm, and appears essential for asexual growth [20]. AP2 domain-containing proteins have evolved as the major regulatory family in the genus Cryptosporidium and C. parvum AP2 domains display reduced binding diversity compared to those of $P$. falciparum [21].

How TFs in Bxj regulate transcription of target genes is still unknown. We identified 21 AP2s, four of which showed high transcription levels by qPCR. Thus, we speculated that these four TFs might play important role in the RBC stage. In this study, we attempt to disrupt these four TFs and confirm the functions of BXIN_0799 in asexual development and RBC invasion of $B x j$. Genes involved in red blood cell invasion, immune evasion, and cellular processes were differentially regulated, when BXIN_0799 gene was disrupted.

\section{Methods}

\section{Plasmid constructs}

To disrupt the BXIN_0799 of AP2 gene family, a plasmid was constructed as presented in Fig. 1a. DNA fragments of ef $1 a-B 5^{\prime}$ non-coding region (ef1a-NR) and rap3' terminal region (rap-TR) were amplified from Bxj genomic DNA, and the blasticidin-S deaminase (BSD) gene was amplified from plasmid pgfp-bsd-ef (kindly gifted by Carlos E. Suarez) (primer sets listed in Table 1). Then, AP2-BSD plasmid was generated by cloning these three fragments into BamH1 site of plasmid pBluescript SK(+) (PBS) using a CloneExpress MultiS One Step Cloning Kit (Vzayme, Nanjing, China) according manufacture's instruction. Homologous upstream and downstream sequences of BXIN_0799 open reading frame (ORF) were amplified from $B \times j$ genomic DNA and inserted into Apa 1 and Sac 1 sites of plasmid AP2-BSD, respectively, which was named as pbs-AP2-M plasmid, confirmed by sequencing and extracted using a QIAGEN Plasmid Maxi Kit (Qiagen, Hilden, Germany) according to the manufacturer's instructions.

\section{Knockout strain of Bxj transcriptional factor BXIN_0799}

Bxj merozoites were cultured in a complete medium with 20\% fetal bovine serum (FBS) (Gibco, Carlsbad, CA, USA) and 7.5\% of fresh sheep RBCs in Roswell Park Memorial Institute 1640 (RPMI-1640) medium (Lonza Biologics, Portsmouth, NH, USA) $[22,23]$. When parasitemia reached to $20 \%$, the culture was collected and washed three times with cold cytomix buffer (120 $\mathrm{mM} \mathrm{KCl}, 0.15 \mathrm{mM} \mathrm{CaCl}_{2}, 10 \mathrm{mM} \mathrm{K}_{2} \mathrm{HPO}_{4} / \mathrm{KH}_{2} \mathrm{PO}_{4}, \mathrm{pH}$ 7.6, $25 \mathrm{mM} \mathrm{HEPES}$. pH7.6, 2 mM EGTA, 5 mM MgCl , final pH 7.6) [24, $25,26,27,28]$. Then, $20 \mu \mathrm{g}$ of circular plasmid of pbs-AP2-M was transfected into $3 \times 10^{8}$ Bxj merozoites with parameters of $1200 \mathrm{~V}$ and $25 \mu \mathrm{F}$ using a Gene Pulser Xcell ${ }^{\mathrm{TM}}$ Electroporation System (Bio-Rad Laboratories, Hercules, CA, USA). Transfected cells were transferred into 24-well culture plate with complete medium (7.5\% fresh sheep RBCs and 20\% FBS in RPMI-1640) and supplied with $10 \mu \mathrm{g} / \mathrm{ml}$ of BSD (Gibco, R21001). When drug resistant parasites were observed by microscopy in thin blood smear stained with Giemsa, a clonal strain (KO-AP2-M), in which BXIN_0799 was knocked out, was obtained by limiting dilution. Briefly, infected RBCs were diluted to 2.5 infected RBCs/mL and $100 \mu \mathrm{l}$ of that was dispensed into each well of 96well culture plate for 17 days. Complete medium was replaced every three days.

\section{RNA-seq analysis}

Using an in vitro Bxj merozoite cultural system, we collected triplicated samples from wild type (WT) and KO-AP2-M parasites for transcriptome analysis. Total RNA was extracted from WT and KO-AP2-M merozoites using TRIZOL method and assessed for quality using an Agilent 2100 Bioanalyzer. mRNA was purified from total RNA using poly-T attached beads. cDNA synthesis was performed using random hexamer primer and M-MLV Reverse Transcriptase. A library of 400 bp paired-end reads was constructed and sequenced using an Illumina Novaseq platform. The data was submitted to China National Center 
for Bioinformation under the accession number CRA005148. Low-quality reads/base and adaptor sequences were removed by Trim Galore (https://github.com/FelixKrueger/TrimGalore). Hisat2 (v2.0.5) was used to map pair-end reads to the reference genome [29, 30]. Fragments per kilobase of transcript sequence per Million base pairs (FPKM) of each gene was calculated based on the length of the gene and reads mapped to a specific gene. Differential expression analysis of WT and KO-AP2-M groups was determined using the DESeq2 R package (1.20.0). P-value of 0.05 and absolute 2 -fold changes were set as the threshold for significantly differential expressions.

The clusterProfiler R package was employed to determine Gene Ontology (GO) enrichment and KEGG pathway analyses of differentially expressed genes. Expected function of these genes were predicted using BLASTP and Interproscan (v5.52-86.0) [31].

\section{Results}

\section{Disruption of Bxj transcriptional factor BXIN_0799 gene}

To determine the functions of AP2 gene family in Bxj development, we attempted to disrupt four of the 21 AP2 genes in $B x j$ genome (BXIN_0799, BXIN_2578, BXIN_2299, BXIN_2747), whose transcriptional levels were relatively high in red blood cell stage. We obtained a KO-AP2-M mutation strain (BXIN_0799 gene was disrupted), but we were unable to generate disrupted strains of the remaining three genes (Fig. 1), suggesting they may play essential roles in asexual development and viability of $B x j$ in erythrocytes stage. Further studies, such as tagging the genes for protein-protein interaction analysis and protein expression analysis, are needed to illustrate the functional roles of these genes. We carefully compared the morphologies of WT and KO-AP2-M in asexual (RBC) stage of merozoites in vitro culture, however, no obvious differences were observed between two groups (data not shown).

\section{Transcriptional regulation of BXIN_0799}

Orthologs of BXIN_0799 can be identified in genomes across piroplasm parasites, such Theileria annulata, T. orientalis, $B$. bovis, $B$. microti. Although the values of ortholog gene size across piroplasm parasites are variable, sequences of predicted AP2 domains of these genes are largely conserved, suggesting a certain degree of functional conservation. To unravel the molecular functions of BXIN_0799, we investigated the transcriptome of KO-AP2-M by high throughout RNA sequencing technology.

We were interested in transcriptional abundant variation of genes regulated by BXIN_0799. When parasitemia reached to $\sim 20 \%$, samples of three biological replicates from each of WT and KO-AP2-M strains were collected for RNA sequencing. Over 40 million cleaned reads were generated from each sample. More than $85 \%$ of paired reads were mapped against the $B \times j$ reference genome. The values of Pearson correlation among biological replicate samples were over 0.97 (Supplementary Fig. 1). Of 3163 identified genes, 282 genes were down regulated in KO-AP2-M compared with WT. 79.9\% of these genes were involved in the biological process by GO enrichment analysis, while the remaining $30.1 \%$ of genes were annotated as uncharacterized protein (Supplement Table 1, Fig. 2 and Fig. 3). We noticed that down-regulated genes palyed critical roles in cellular process, including transcription (Translation initiation factor IF-2, Helicase and RNA polymerase) and posttranslational modification (Palmitoyltransferase, Serine/threonine-specific protein phosphatase and Protein kinase).

\section{BXIN_0799 regulates genes involved in asexual development and immune evasion of Bxj}

Comparative analyses of inhibitory efficiency of disrupted and WT strains showed that a mild degree of growth inhibitory was observed in KO-AP2-M strains (Fig. 4). Some genes, classified as TFs, contributed to host cell invasion and facilitated immune evasion, were down regulated in BXIN_0799 disrupted strain. Transcription levels of transcriptional factors, such as BXIN_2860, BXIN_2746, BXIN_1453 and BXIN_2299, were also positively regulated by BXIN_0799. Variant erythrocyte surface antigen (VESA) is well known to be exported to the surface of infected erythrocytes, which is an efficient mean of evading the humoral immune response and facilitated diversity of parasite ligand to bind different receptors on hose cell. We observed decreased transcription levels for ten VESA genes in KO-AP2-M strain: BXIN_1817, BXIN_2430, BXIN_0930, BXIN_2830, 
BXIN_2220, BXIN_1601, BXIN_0957, BXIN_1534, BXIN_2426, BXIN_2425, BXIN_2429, BXIN_1820, BXIN_1890, BXIN_1821, BXIN_0275 and BXIN_1599 (Supplement Table 1).

Rhoptries are cellular organelles of apicomplexan parasites. During the invasion of a host cell, a parasite's rhoptries release proteins that bind to host cell receptors. Rhoptry-associated protein 1 (RAP1) has been intensively investigated in apicomplexan parasites; it has a long time been considered a good candidate antigen for a vaccine. We also determined that transcription levels of RAP1 gene was significantly decreased in KO-AP2-M strain compared with WT.

\section{Discussion}

AP2 gene family performs multiple functions in Apicomplexan parasite asexual proliferation, sexual reproduction, gametogenesis, and sporogony [32, 33, 34]. In P. falciparum, AP2-I induces chromatin remodeling and recruits the transcriptional machinery to regulate invasion [17]. Activating gametocyte specific genes, AP2-G regulates sexual reproduction and suppresses asexual gene expression [19]. Gametocyte-specific genes that are specifically expressed in gametogenesis stage are regulated by AP2-0, posing a direct influence on ookinete motility [33, 35]. AP2-Sp is involved in sporozoite formation in the oocyst [36, 37]. These AP2s genes are conserved across Plasmodium spp., but not in Piroplasm species. In piroplasm parasites, AP2 genes haven been investigated in phylogenetic analysis; their functions in parasite life cycles are limited. Similarly, homologous genes of BXIN_0799 have been identified in the genomes of Babesia ovata (XP_028868794), $B$. bovis (XP_001609401), B. bigemina (XP_012770144), T. orientalis (PVC59920 and PVC55584) and T. annulata (XP_955102) using BLASTP alignment program and comparative genomic analysis [38, 39]. However, there are no reports on the related functions of the BXIN_0799 and its homologous genes. We identified four TFs that showed higher transcriptional level in erythrocytic stages by real time PCR. Homologous recombination technique was used to knock out these TFs [7]. Unfortunately, we were only able to disrupt one of these genes (BXIN_0799); disruption of the other three TFs was not successful even after three attempts, suggesting they have essential functions in the RBC stage.

In KO-AP2-M, thirty-four of functional protein were corded by down-regulated genes, associated with regulation of transcription, translation, and protein-modification. Bromodomains (BXIN_0081, BXIN_0164) contain protein interaction modules that exclusively recognize acetylation motifs and play an important role in transcriptional activation [40,41]. This domain is involved in the regulation of transcription, in particular acetylating histones, remodeling chromatin, and recruiting other factors for transcription [42]. Helicases perform critical roles both in various biological pathways and nucleic acid metabolism. Parasite-specific helicases and C-terminal of helicases that play an important role in unwinding partially duplex DNA and RNA substrates of $P$. falciparum [43]. A recent study result showed that the serine/threonine-specific protein phosphatase (STP) was important for crucial dephosphorylation events, cell signaling, and the ability of parasite to adapt to hosts in different Leishmania species [44]. DHHC (aspartate-histidine-histidine-cysteine) palmitoyltransferases, eukaryotic integral membrane enzymes, catalyze protein palmitoylation, which has correlated with small guanosine triphosphatase (GTPase) signaling and cell adhesion [45]. In mammals, DHHC palmitoyltransferases promote ClipR-59 palmitoylation and regulate interaction of ClipR-59 and plasma membrane [46, 47]. The specific mechanism of DHHC in Babesia spp. is not clear.

The four TFs (BXIN_2299, BXIN_1453, BXIN_2746, BXIN_2860) were also observed down-regulation in KO-AP2-M. Through the gene knock out and transcriptome data we can't confirm whether the transcriptional level of these genes was directly or indirectly controlled by BXIN_0799. Further study is needed to investigate that the regulation of BXIN_0799 and BXIN_2299, BXIN_1453, BXIN_2746, and BXIN_2860.

Erythrocyte invasion, erythrocyte adhesion, immune evasion associated genes, such as VESA1, Rhomboid-like superfamily (BXIN_0048) and Rhoptry-associated protein 1 (BXIN_2991), are down-regulated at the transcription level in KO-AP2-M. VESA1 that facilitated parasite escaping host immune responses, mediates antigenic variation, and plays a role in parasite development [48]. Each erythrocytic cycle stages express one type of VESA, which may vary widely depending on developmental stages in specific species $[9,49]$. Whether these down regulated VESA genes are expressed at the same stage is needed to further confirmation, as bulk RNA sequencing samples were collected at the red blood cell stage. They may

Page 5/11 
contain female and male gametocytes. Some of these VESA genes may specifically express at the stage of gametocyte. Our results showed that down regulated VESA posed no significant influence on asexual development, thus we speculated that these VESAs might perform a function in other stages, such as sexual reproduction or the sporozoites development. This phenomenon could also be explained that slight positive correlation in protein expression and transcriptional level of VESA1. In addition, AP2-I is responsible for regulating RBC invasion in P. falciparum and associates with the promoters of invasionrelated genes [17]. It seems that BXIN_0799 and AP2-I performed similar functions, although they shared low sequence similarity.

Rhomboid-like superfamily has been widely identified across apicomplexan parasites and regulates membrane proteins through influencing their proteolysis, trafficking, or degradation [50,51]. Rhomboid proteases play important roles in apicomplexan parasites adhesion and invasion $[52,53]$. Till now, the functions of rhomboid proteases have not been extensively studied in Babesia. The unusual enzymology of rhomboid proteases could be a potential target for exploring drugs against babesiosis.

Rhoptry-associated proteins (RAP), immunosuppressive factors released into the host cell, have been recognized as key molecules for parasite invasion and replication in host cells to mediate immunity in the stage of parasitophorous vacuole (PV) formation. RAP-1 is considered as a potential vaccine candidate [54, 55].

Inner membrane complex protein (BXIN_1891, BXIN_2490) might participant in parasite motility and invasion. Inner membrane complex (IMC) of Apicomplexans is a defining feature composed of lipid and protein (gliding-associated potein: GAP 40, GAP45, GAP50) and apparatus of gliding motility and invasion $[56,57,58]$. In addition, the IMC located to very early daughter buds influenced the replication of the Toxoplasma gondii [59].

\section{Conclusions}

In this study, we illustrate the regulation function of BXIN_0799 in Bxj asexual development. Hundreds of genes related to genes from transcription, protein translation, post modification, and even immune response were down-regulated in BXIN_0799 knockout strain. Among these genes, VESA1, RAP1 and TFs are well-known to play critical roles in the processes of immune evasion/host cell invasion of the parasite. A slight influence was observed in RBC stage development. 95 downregulated genes code unknown functional proteins. Whether these products contribute to gametocytogenesis in the RBC stage, sexual development, and even blocking transmission is need to further study.

\section{Declarations}

\section{Acknowledgements}

We acknowledged Carlos E. Suarez from Department of Veterinary Microbiology and Pathology, Washington State University) for technical supports and providing pgfp-bsd-efp and pBluescript SK(+) plasmid. The authors are grateful to Shin-ichiro Kawazu from National Research Center for Protozoan Diseases, Obihiro University of Agriculture and Veterinary Medicine, Obihiro, Hokkaido 080-8555, Japan for providing DHFR-gfp plasmid. We also acknowledged Yijun Chai (Lanzhou Veterinary Research Institute, Chinese Academy of Agricultural Sciences) who contribute to drafts of the introduction and discussion of this manuscript.

\section{Ethics approval and consent to participate}

The collection and manipulation of sheep blood samples was approved by the Animal Ethics Committee of the Lanzhou Veterinary Research Institute, Chinese Academy of Agricultural Sciences. All the procedures conducted were according to the Ethical Procedures and Guidelines of the People's Republic of China.

\section{Competing interests}


The authors declare that they have no competing interests.

\section{Consent for publication}

Not applicable.

\section{Availability of data and materials}

Not applicable.

\section{Funding}

This study was financially supported by the National Science Foundation of China (grant no. 31972701), ASTIP (grant no. CAAS-ASTIP-2016-LVRI), NBCIS (grant no. CARS-37), and the Jiangsu Co-innovation Center Program for the Prevention and Control of Important Animal Infectious Disease and Zoonoses.

\section{Authors' contributions}

Manuscript, JM and YC; reagents/materials/analysis, YL; supervision, JL, YH and GG. All authors have read and approved the final manuscript.

\section{References}

1. Woods K, Perry C, Bruhlmann F, Olias P. Theileria's Strategies and Effector Mechanisms for Host Cell Transformation: From Invasion to Immortalization. Front Cell Dev Biol. 2021;9:662805.

2. Chen M, Liu Q, Xue J, Chen S, Huang D, Yu Y, et al. Spreading of Human Babesiosis in China: Current Epidemiological Status and Future Challenges. China CDC Wkly. 2020;2 33:634-7.

3. Baum J, Richard D, Healer J, Rug M, Krnajski Z, Gilberger TW, et al. A conserved molecular motor drives cell invasion and gliding motility across malaria life cycle stages and other apicomplexan parasites. J Biol Chem. 2006;281 8:5197-208.

4. Vannier EG, Diuk-Wasser MA, Ben Mamoun C, Krause PJ. Babesiosis Infect Dis Clin North Am. 2015;29(2):357-70.

5. Madison-Antenucci S, Wormser GP, Levin AE, Wong SJ. Frequency and magnitude of seroreactivity to Babesia microti in 245 patients diagnosed by PCR in New York State. Diagn Microbiol Infect Dis. 2020;97 1:115008.

6. Guan G, Ma M, Moreau E, Liu J, Lu B, Bai Q, et al. A new ovine Babesia species transmitted by Hyalomma anatolicum anatolicum. Exp Parasitol. 2009;122 4:261-7.

7. Wang J, Wang X, Guan G, Yang J, Liu J, Liu A, et al. Stable transfection system for Babesia sp. Xinjiang Parasit Vectors. 2021;14(1):463.

8. Niu Q, Liu Z, Yang J, Gao S, Pan Y, Guan G, et al. Genetic characterization and molecular survey of Babesia sp. Xinjiang infection in small ruminants and ixodid ticks in China. Infect Genet Evol. 2017;49:330-5.

9. Brayton KA, Lau AO, Herndon DR, Hannick L, Kappmeyer LS, Berens SJ, et al. Genome sequence of Babesia bovis and comparative analysis of apicomplexan hemoprotozoa. Plos Pathog. 2007;3 10:1401-13.

10. Cornillot E, Hadj-Kaddour K, Dassouli A, Noel B, Ranwez V, Vacherie B, et al. Sequencing of the smallest Apicomplexan genome from the human pathogen Babesia microti. Nucleic Acids Res. 2012;40 18:9102-14.

11. Guan G, Korhonen PK, Young ND, Koehler AV, Wang T, Li Y, et al. Genomic resources for a unique, low-virulence Babesia taxon from China. Parasit Vectors. 2016;9 1:564.

12. Coulson RM, Hall N, Ouzounis CA. Comparative genomics of transcriptional control in the human malaria parasite Plasmodium falciparum. Genome Res. 2004;14:8:1548-54.

13. Templeton TJ, lyer LM, Anantharaman V, Enomoto S, Abrahante JE, Subramanian GM, et al. Comparative analysis of apicomplexa and genomic diversity in eukaryotes. Genome Res. 2004;14 9:1686-95. 
14. Balaji S, Babu MM, lyer LM, Aravind L. Discovery of the principal specific transcription factors of Apicomplexa and their implication for the evolution of the AP2-integrase DNA binding domains. Nucleic Acids Res. 2005;33 13:3994-4006.

15. Alzan HF, Knowles DP, Suarez CE. Comparative Bioinformatics Analysis of Transcription Factor Genes Indicates Conservation of Key Regulatory Domains among Babesia bovis, Babesia microti, and Theileria equi. PLoS Negl Trop Dis. 2016;10 11:e0004983.

16. Carrington E, Cooijmans RHM, Keller D, Toenhake CG, Bartfai R, Voss TS. The ApiAP2 factor PfAP2-HC is an integral component of heterochromatin in the malaria parasite Plasmodium falciparum. iScience. 2021;24 5:102444.

17. Santos JM, Josling G, Ross P, Joshi P, Orchard L, Campbell T, et al. Red Blood Cell Invasion by the Malaria Parasite Is Coordinated by the PfAP2-I Transcription Factor. Cell Host Microbe. 2017;21 6:731-41 e10.

18. Kafsack BF, Rovira-Graells N, Clark TG, Bancells C, Crowley VM, Campino SG, et al. A transcriptional switch underlies commitment to sexual development in malaria parasites. Nature. 2014;507 7491:248-52.

19. Josling GA, Russell TJ, Venezia J, Orchard L, van Biljon R, Painter HJ, et al. Dissecting the role of PfAP2-G in malaria gametocytogenesis. Nat Commun. 2020;11 1:1503.

20. Martins RM, Macpherson CR, Claes A, Scheidig-Benatar C, Sakamoto H, Yam XY, et al. An ApiAP2 member regulates expression of clonally variant genes of the human malaria parasite. Plasmodium falciparum Sci Rep. 2017;7(1):14042.

21. Oberstaller J, Pumpalova Y, Schieler A, Llinas M, Kissinger JC. The Cryptosporidium parvum ApiAP2 gene family: insights into the evolution of apicomplexan AP2 regulatory systems. Nucleic Acids Res. 2014;42 13:8271-84.

22. Guan G, Ma M, Liu A, Ren Q, Wang J, Yang J, et al. A recently identified ovine Babesia in China: serology and seroepidemiology. Parasitol Int. 2012;61 4:532-7.

23. Guan G, Ma M, Liu A, Du P, Ren Q, Li Y, et al. Continuous in vitro cultivation of a recently identified Babesia that infects small ruminants in China. Vet Parasitol. 2012;187(3-4):371-8.

24. Suarez CE, McElwain TF. Transient transfection of purified Babesia bovis merozoites. Exp Parasitol. 2008;118 4:498-504.

25. Suarez CE, McElwain TF. Stable expression of a GFP-BSD fusion protein in Babesia bovis merozoites. Int J Parasitol. 2009;39(3):289-97.

26. Asada M, Tanaka M, Goto Y, Yokoyama N, Inoue N, Kawazu S. Stable expression of green fluorescent protein and targeted disruption of thioredoxin peroxidase-1 gene in Babesia bovis with the WR99210/dhfr selection system. Mol Biochem Parasitol. 2012;181 2:162-70.

27. Hakimi H, Yamagishi J, Kegawa Y, Kaneko O, Kawazu S, Asada M. Establishment of transient and stable transfection systems for Babesia ovata. Parasit Vectors. 2016;9:171.

28. Liu M, Adjou Moumouni PF, Asada M, Hakimi H, Masatani T, Vudriko P, et al. Establishment of a stable transfection system for genetic manipulation of Babesia gibsoni. Parasit Vectors. 2018;11 1:260.

29. Kim D, Langmead B, Salzberg SL. HISAT: a fast spliced aligner with low memory requirements. Nat Methods. 2015;12 4:357-60.

30. Kim D, Paggi JM, Park C, Bennett C, Salzberg SL. Graph-based genome alignment and genotyping with HISAT2 and HISAT-genotype. Nat Biotechnol. 2019;37 8:907-15.

31. Quevillon E, Silventoinen V, Pillai S, Harte N, Mulder N, Apweiler R, et al. InterProScan: protein domains identifier. Nucleic Acids Res. 2005;33 Web Server issue:W116-20.

32. Bennink S, Kiesow MJ, Pradel G. The development of malaria parasites in the mosquito midgut. Cell Microbiol. 2016;18 7:905-18.

33. Zhang C, Li Z, Cui H, Jiang Y, Yang Z, Wang X, et al. Systematic CRISPR-Cas9-Mediated Modifications of Plasmodium yoelii ApiAP2 Genes Reveal Functional Insights into Parasite Development. mBio. 2017;8 6.

34. Gomez-Diaz E, Yerbanga RS, Lefevre T, Cohuet A, Rowley MJ, Ouedraogo JB, et al. Epigenetic regulation of Plasmodium falciparum clonally variant gene expression during development in Anopheles gambiae. Sci Rep. 2017;7:40655. 
35. Modrzynska K, Pfander C, Chappell L, Yu L, Suarez C, Dundas K, et al. A Knockout Screen of ApiAP2 Genes Reveals Networks of Interacting Transcriptional Regulators Controlling the Plasmodium Life Cycle. Cell Host Microbe. 2017;21(1):11-22.

36. Jeninga MD, Quinn JE, Petter M. ApiAP2 Transcription Factors in Apicomplexan Parasites. Pathogens. 2019;8:2.

37. Yuda M, Iwanaga S, Shigenobu S, Kato T, Kaneko I. Transcription factor AP2-Sp and its target genes in malarial sporozoites. Mol Microbiol. 2010;75 4:854-63.

38. Yamagishi J, Asada M, Hakimi H, Tanaka TQ, Sugimoto C, Kawazu SI. Whole-genome assembly of Babesia ovata and comparative genomics between closely related pathogens. BMC Genom. 2017;18:1:832.

39. Jaimes-Duenez J, Triana-Chavez O, Holguin-Rocha A, Tobon-Castano A, Mejia-Jaramillo AM. Molecular surveillance and phylogenetic traits of Babesia bigemina and Babesia bovis in cattle (Bos taurus) and water buffaloes (Bubalus bubalis) from Colombia. Parasit Vectors. 2018;11:1:510.

40. Filippakopoulos P, Picaud S, Mangos M, Keates T, Lambert JP, Barsyte-Lovejoy D, et al. Histone recognition and largescale structural analysis of the human bromodomain family. Cell. 2012;149(1):214-31.

41. Wang N, Wu R, Tang D, Kang R. The BET family in immunity and disease. Signal Transduct Target Ther. 2021;6(1):23.

42. Josling GA, Selvarajah SA, Petter M, Duffy MF. The role of bromodomain proteins in regulating gene expression. Genes (Basel). 2012;3(2):320-43.

43. Chauhan M, Sourabh S, Yasmin R, Pahuja I, Tuteja R. Biochemical characterization of Plasmodium falciparum parasite specific helicase 1 (PfPSH1). FEBS Open Bio. 2019;9:11:1909-27.

44. Freitas-Mesquita AL, Dos-Santos ALA, Meyer-Fernandes JR. Involvement of Leishmania Phosphatases in Parasite Biology and Pathogeny. Front Cell Infect Microbiol. 2021;11:633146.

45. Rana MS, Kumar P, Lee CJ, Verardi R, Rajashankar KR, Banerjee A. Fatty acyl recognition and transfer by an integral membrane S-acyltransferase. Science. 2018;359:6372.

46. Ren W, Sun Y, Du K. DHHC17 palmitoylates ClipR-59 and modulates ClipR-59 association with the plasma membrane. Mol Cell Biol. 2013;33 21:4255-65.

47. Nadolski MJ, Linder ME. Protein lipidation. FEBS J. 2007;274 20:5202-10.

48. Wang X, Xiao YP, Bouchut A, Al-Khedery B, Wang H, Allred DR. Characterization of the unusual bidirectional ves promoters driving VESA1 expression and associated with antigenic variation in Babesia bovis. Eukaryot Cell. 2012;11 3:260-9.

49. Nie Z, Ao Y, Wang S, Shu X, Li M, Zhan X, et al. Erythrocyte Adhesion of Merozoite Surface Antigen 2c1 Expressed During Extracellular Stages of Babesia orientalis. Front Immunol. 2021;12:623492.

50. Freeman M. The rhomboid-like superfamily: molecular mechanisms and biological roles. Annu Rev Cell Dev Biol. 2014;30:235-54.

51. Ticha A, Collis B, Strisovsky K. The Rhomboid Superfamily: Structural Mechanisms and Chemical Biology Opportunities. Trends Biochem Sci. 2018;43 9:726-39.

52. Urban S, Freeman M. Substrate specificity of rhomboid intramembrane proteases is governed by helix-breaking residues in the substrate transmembrane domain. Mol Cell. 2003;11 6:1425-34.

53. Sibley LD. The roles of intramembrane proteases in protozoan parasites. Biochim Biophys Acta. 2013;1828 12:2908-15.

54. Ito D, Hasegawa T, Miura K, Yamasaki T, Arumugam TU, Thongkukiatkul A, et al. RALP1 is a rhoptry neck erythrocytebinding protein of Plasmodium falciparum merozoites and a potential blood-stage vaccine candidate antigen. Infect Immun. 2013;81 11:4290-8.

55. Liffner B, Frolich S, Heinemann GK, Liu B, Ralph SA, Dixon MWA, et al. PfCERLI1 is a conserved rhoptry associated protein essential for Plasmodium falciparum merozoite invasion of erythrocytes. Nat Commun. 2020;11 1:1411.

56. Gilk SD, Gaskins E, Ward GE, Beckers CJ. GAP45 phosphorylation controls assembly of the Toxoplasma myosin XIV complex. Eukaryot Cell. 2009;8(2):190-6. 
57. Fauquenoy S, Hovasse A, Sloves PJ, Morelle W, Dilezitoko Alayi T, Slomianny C, et al. Unusual N-glycan structures required for trafficking Toxoplasma gondii GAP50 to the inner membrane complex regulate host cell entry through parasite motility. Mol Cell Proteomics. 2011;10 9:M111 008953.

58. Kumar V, Behl A, Kapoor P, Nayak B, Singh G, Singh AP, et al. Inner membrane complex 1 l protein of Plasmodium falciparum links membrane lipids with cytoskeletal element 'actin' and its associated motor 'myosin'. Int J Biol Macromol. 2019;126:673-84.

59. Torres JA, Pasquarelli RR, Back PS, Moon AS, Bradley PJ. Identification and Molecular Dissection of IMC32, a Conserved Toxoplasma Inner Membrane Complex Protein That Is Essential for Parasite Replication. mBio. 2021;12:1.

\section{Tables}

Table 1. Primer sequences for knockout transcription factor BXIN_0799 and identification of clonal strain.

\begin{tabular}{|c|c|c|c|}
\hline $\begin{array}{l}\text { Gene } \\
\text { name }\end{array}$ & Sequence $₫ 5^{\prime}-3^{\prime} \rrbracket$ & $\begin{array}{l}\text { Size of } \\
\text { target } \\
\text { sequence } \\
\text { (bp) }\end{array}$ & $\begin{array}{l}\text { Amplified } \\
\text { fragment }\end{array}$ \\
\hline $\begin{array}{l}\text { ef1a- } \\
\text { NR-F }\end{array}$ & TATCGAATTCCTGCAGCCCGGGGGATCCGTTGTCTTTTGGTATTACATAATGTATTGC & 958 & $\begin{array}{l}\text { ef1a-B } \\
\text { promoter } \\
\text { sequence }\end{array}$ \\
\hline $\begin{array}{l}\text { ef1a- } \\
\text { NR-R }\end{array}$ & TCTTCTTGAGACAAAGGCTTGGCCATTTTCGTGGAGTTTTAACTAGAGTCTAACT & & \\
\hline bsd-F & ATGGCCAAGCCTTTGTCTCA & 396 & bad gene \\
\hline bsd-R & TTAGCCCTCCCACACATAAC & & \\
\hline $\begin{array}{l}\text { rap-TR- } \\
\mathrm{F}\end{array}$ & GGGAGGGCTAAGCGGCCGCGGCAGCTTCAAAGCG & 1369 & rap3' TR \\
\hline $\begin{array}{l}\text { rap-TR- } \\
\text { R }\end{array}$ & GCGGCCGCTCTAGAACTAGTGGATCCCTTAACACCAGGCAAT & & \\
\hline $\begin{array}{l}\text { BXAP2- } \\
\text { M -5' } \\
\text { UTR-F }\end{array}$ & AGGGCGAATTGGGTACCGGGCCCGTCGTGCCCATCTTATTTGCG & 920 & $\begin{array}{l}\text { upstream } \\
\text { sequence of } \\
\text { BxAP2- } \\
\text { M ORF }\end{array}$ \\
\hline $\begin{array}{l}\text { BXAP2- } \\
\text { M-5' } \\
\text { UTR -R }\end{array}$ & GATACCGTCGACCTCGAGGGGGGGCCCCGGCCAATTCCATGTGAACA & & \\
\hline $\begin{array}{l}\text { BxAP2- } \\
\text { M -3' } \\
\text { UTR-F }\end{array}$ & AGCGGCCGCCACCGCGGTGGAGCTCGCTAGTTGCGTTGAGTAAGCCA & 933 & $\begin{array}{l}\text { downstream } \\
\text { sequence of } \\
\text { BxAP2-M } \\
\text { ORF }\end{array}$ \\
\hline $\begin{array}{l}\text { BXAP2- } \\
\text { M-3' } \\
\text { UTR -R }\end{array}$ & CACTAAAGGGAACAAAAGCTGGAGCTCTGGGAATGAAGTACGTTGTAAACTG & & \\
\hline F1-F & GCCAAGCCTTTGTCTCAAGAAG & 7394 & $\mathrm{~F} 1$ \\
\hline F1-R & CTAGCAGTGTGTTAGCGTGC & & \\
\hline
\end{tabular}

Homologous sequences are underlined and are employed to connect with upstream and downstream genes.

\section{Figures}




\section{Figure 1}

Disruption of $B x j$ BXIN_0799. AP2-BSD shows the structure of the plasmid used as the backbone to generate the BXIN_0799 knockout plasmid (pbs-AP2-M). The BXIN_0799 locus illustrates the organization of BXIN_0799 in the Bxj genome. Mutant Bxj parasite lines are as in the KO-AP2-M diagram.

\section{Figure 2}

GO enrichment analysis of the downregulated genes in KO-AP2-M.

\section{Figure 3}

KEGG analysis of the downregulated genes in KO-AP2-M.

\section{Figure 4}

Growth inhibition following disruption of BXIN_0799 in KO-AP2-M compared with WT.

\section{Supplementary Files}

This is a list of supplementary files associated with this preprint. Click to download.

- Graphicalabstract.docx

- SupplementFig.1.tif

- SupplementaryTable1.xls 